\title{
Association of Breastfeeding and the Federal Poverty Level: National Survey of Family Growth, 2011-2013
}

\author{
R. Constance Wiener, ${ }^{1}$ Usha Sambamoorthi, ${ }^{2}$ Sarah E. Hayes, ${ }^{3}$ and Ilana R. Azulay Chertok ${ }^{4}$ \\ ${ }^{1}$ Department of Dental Practice and Rural Health, School of Dentistry, West Virginia University, 104A Health Sciences Center Addition, \\ P.O. Box 9448, Morgantown, WV 26506, USA \\ ${ }^{2}$ Department of Pharmaceutical Systems and Policy, West Virginia University School of Pharmacy, \\ Robert C. Byrd Health Sciences Center (North), P.O. Box 9510, Morgantown, WV 26506-9510, USA \\ ${ }^{3}$ West Virginia University Department of Psychology, 53 Campus Drive, P.O. Box 6040, Morgantown, WV 26506, USA \\ ${ }^{4}$ University of North Carolina Charlotte School of Nursing, CHHS-420, Charlotte, NC 28223, USA \\ Correspondence should be addressed to R. Constance Wiener; rwiener2@hsc.wvu.edu
}

Received 1 December 2015; Revised 29 January 2016; Accepted 15 February 2016

Academic Editor: Suminori Kono

Copyright @ 2016 R. Constance Wiener et al. This is an open access article distributed under the Creative Commons Attribution License, which permits unrestricted use, distribution, and reproduction in any medium, provided the original work is properly cited.

Breastfeeding is strongly endorsed in the Healthy People 2020 goals; however, there remain many disparities in breastfeeding prevalence. The purpose of this study was to examine the association between breastfeeding and the Federal Poverty Level in the United States. Data from 5,397 women in the National Survey of Family Growth 2011-2013 survey were included in this study. The data were analyzed for descriptive features and logistic regressions of the Federal Poverty Level on breastfeeding. There were $64.1 \%$ of women who reported breastfeeding. Over one-third (35.2\%) of women reported having a household income of 0-99\% of the Federal Poverty Level. There were $15.2 \%$ of women who reported an income of $400 \%$ and above the Federal Poverty Level. With statistical adjustment for maternal age, race/ethnicity, education, marital status, parity, preterm birth, birth weight, insurance, and dwelling, the Federal Poverty Level was not significantly associated with breastfeeding. In this recent survey of mothers, Federal Poverty Level was not shown to be a significant factor in breastfeeding.

\section{Introduction}

The American Academy of Pediatrics recommends the exclusive breastfeeding of infants to age six months, with continued breastfeeding (complemented by solid foods) for one year or longer [1]. The United States (US) Department of Health and Human Services recognizes the public health benefits of breastfeeding and has nine breastfeeding-related objectives for Healthy People 2020 goals [2]. These objectives include increasing the number of infants having ever been breastfed from the baseline of $74.0 \%$ to $81.9 \%$; increasing the number of infants who are breastfed to age 6 months from the baseline of $43.5 \%$ to $60.6 \%$; and increasing the number of facilities that provide recommended care for lactating mothers and newborns from a baseline of $2.9 \%$ to $8.1 \%$ [2].

There are many barriers to breastfeeding that have been reported in earlier studies including lack of support $[3,4]$, public beliefs [3], difficulty with the breast pump [5], young age of mother, less education, unmarried status, fear of embarrassment, fear of being fired, privacy, sexualization of the breast, change in appearance of the breast, pain, bleeding, difficulty latching-on, insufficient milk, race/ethnicity, and low income [6]. In a population-based study examining the influence of poverty and participation in the federal Special Supplemental Nutrition Program for Women, Infants, and Children (WIC) in South Carolina, researchers found that WIC participation was the strongest predictor of lack of breastfeeding initiation in that state [7].

Women who participated in WIC programs faced additional barriers to breastfeeding [8]. One of the themes that emerged in a qualitative study of WIC counselors serving primarily African American families was that formula use was seen as a sign of wealth [9]. Prior to the recently revised WIC breastfeeding incentive program of augmented 
food packages for breastfeeding women, WIC participation had been associated with lower breastfeeding initiation and duration rates [10]. WIC credits can be used for supplemental formula, and many clients viewed the supplemental formula as more valuable than the offset of expanded food packages [8].

With goals in place and concerted efforts to increase breastfeeding rates, research results have been inconsistent regarding the association between family income and breastfeeding; some researchers indicate no association [11, 12], others support an association [13, 14], and others report equivocal results [15]. The aim of this study was to determine if there was an association between breastfeeding and the Federal Poverty Level (FPL) using data from the National Survey of Family Growth 2011-2013.

\section{Methods and Materials}

Data from the National Survey of Family Growth (NSFG) 2011-2013 data were used to conduct a cross-sectional secondary data analysis of the association of FPL and breastfeeding. The 2011-2013 survey is the NSFG's 8th data file release since 1973 (National Survey of Family Growth 2015) [16]. The NSFG survey was specifically designed to determine family trends as well as differences among groups in family sizes, family structure, use of contraception, sexual activity, and infertility for use in designing health services and educational programs [16]. The sampling was a multistage probability-based national representative of US households [16]. Details of the survey are provided at the NSFG website, http://www.cdc.gov/nchs/nsfg/nsfg_2011_2013_puf.htm.

This study received the West Virginia University Institutional Review Board study acknowledgement (protocol number 1502572781). The research was conducted in accordance with prevailing ethical principles.

2.1. Study Population. Participants in the current study of the association between breastfeeding and FPL were women of childbearing age (14 to 44 years) who had completed NSFG 2011-2013 data for the following variables: breastfeeding; FPL status; and race/ethnicity. The sample size was 5,397 women.

2.2. Variable Definitions. The dependent variable was breastfeeding, defined as breastfeeding one week or more. (The definition did not include intent to breastfeed.) The variable was a dichotomized "yes" or "no" variable.

The independent variable was the FPL of the mother. FPL was provided by the NSFG as a recoded variable with five levels: 0-99\% FPL, 100-199\% FPL, 200-299\% FPL, 300-399\% FPL, and $400 \%$ and above FPL. Other sociodemographic and health variables included in the analyses were maternal age (14 to less than 20 years; 20 to less than 25 years; 25 to less than 30 years; or 30 to 44 years); maternal race/ethnicity (nonHispanic white; non-Hispanic black; or Hispanic); maternal education (less than high school; high school graduate; some higher education; or Associate degree and above); marital status (married or single/divorced/separated/widowed); parity (first infant or 2 or more); preterm birth (yes, less than 37 weeks, or no, 37 weeks and above); low birth weight (yes, less than 2,500 grams; no, 2,500 grams and above); insurance (private or MediGap; Medicaid, CHIP, or a state-sponsored plan; Medicare, military, or other government plan; or single service, Indian Health Service, or not covered); and dwelling (urban, principal city; urban, other than principal city; or rural).

2.3. Analyses. Due to the complex sampling design of the NSFG 2011-2013, analyses were conducted to account for the computational units, strata, and final weights. SAS version $9.3^{\circ}$ (Cary, NC) software was used to determine the descriptive characteristics of the sample, and the relationship between breastfeeding and FPL in logistic regression. The model-based imputations in the NSFG 2011-2013 data for the variables chosen had a maximum imputation occurring with FPL (5.94\%). An adjusted regression model was built which included maternal age, maternal race/ethnicity; maternal education; marital status; parity; preterm birth, birth weight; insurance; and dwelling.

\section{Results}

The study population was derived from the National Survey of Family Growth 2011-2013 data. There were 5,397 participants in this study. There were 3,302 (64.1\%) participants who breastfed and 2,095 (35.9\%) who did not breastfeed. There were $2,406(35.3 \%)$ at $0-99 \%$ of the FPL; $1,324(23.3 \%)$ at the $100-199 \%$ FPL; 701 (15.9\%) at the 200-299\% FPL; 414 (10.4\%) at the $300-399 \%$ FPL; and $552(15.1 \%)$ at or above the $400 \%$ FPL. The sample description is presented in Table 1.

In unadjusted logistic analysis, there were significant relationships between breastfeeding and income for all of the participants. In stratified analyses, there were significant associations between breastfeeding and income with maternal age in the 25-30 year category; maternal age in the 30 year and older category; maternal race/ethnicity; maternal education in the Associate degree and above category; parity in the second baby or above category; preterm birth; insurance in all categories except the Medicaid, Chip, State sponsored plan category; and urban dwelling. The results of the unadjusted logistic regressions are presented in Table 2.

The results of two logistic regression models on breastfeeding are presented in Table 3. The first model demonstrates the combined effect of entering maternal age, maternal education, marital status, and dwelling on the relationship between breastfeeding and FPL. FPL is attenuated and no longer significant in this parsimonious model. The complete model further attenuated the relationship.

There were no interactions with FPL and marital status, preterm birth, and dwelling when each variable was entered individually in the logistic regressions. There was a slight attenuation of the association of FPL and breastfeeding when maternal age and maternal education were entered individually in logistic regressions. It was the combined effects of the variables which altered the FPL-breastfeeding relationship. The results of the adjusted logistic regression analyses stratified for each category of maternal race/ethnicity, marital status, and preterm birth are presented in Table 4. 
TABLE 1: Descriptive statistics of participants in the National Survey of Family Growth (NSFG) 2011-2013.

\begin{tabular}{|c|c|c|c|c|c|}
\hline & \multicolumn{2}{|c|}{ Yes breastfeeding } & \multicolumn{2}{|c|}{ No breastfeeding } & \multirow{2}{*}{$\begin{array}{l}\text { Total } \\
\text { Total }\end{array}$} \\
\hline & $N$ & Wt Col\% & $N$ & Wt Col\% & \\
\hline All & 3,302 & 64.1 & 2,095 & 35.9 & 5,397 \\
\hline \multicolumn{6}{|l|}{ Federal Poverty Level (FPL) } \\
\hline $0-99 \%$ of FPL & 1,270 & 18.9 & 1,136 & 16.3 & 2,406 \\
\hline 100-199\% FPL & 811 & 14.7 & 513 & 8.6 & 1,324 \\
\hline 200-299\% FPL & 487 & 10.9 & 214 & 5.0 & 701 \\
\hline $300-399 \%$ FPL & 299 & 7.4 & 115 & 3.0 & 414 \\
\hline $400 \%$ and above FPL & 435 & 12.2 & 117 & 3.0 & 552 \\
\hline Maternal age (all) & 3,302 & 64.1 & 2,095 & 35.9 & 5,397 \\
\hline 14 to less than 20 years & 472 & 6.6 & 468 & 7.2 & 940 \\
\hline 20 to less than 25 years & 1,065 & 18.1 & 827 & 13.2 & 1,892 \\
\hline 25 to less than 30 years & 995 & 21.2 & 504 & 10.0 & 1,499 \\
\hline 30 years to 44 years & 770 & 18.3 & 296 & 5.4 & 1,066 \\
\hline Maternal race/ethnicity (all) & 3,302 & 64.1 & 2,095 & 35.9 & 5,397 \\
\hline Non-Hispanic white & 1,535 & 39.7 & 802 & 19.3 & 2,337 \\
\hline Non-Hispanic black & 561 & 7.1 & 752 & 8.6 & 1,313 \\
\hline Hispanic & 1,206 & 17.3 & 541 & 7.9 & 1,747 \\
\hline Maternal education (all) & 3,302 & 64.1 & 2,095 & 35.9 & 5,397 \\
\hline Less than high school & 639 & 10.6 & 513 & 6.6 & 1,152 \\
\hline High school graduate & 828 & 14.4 & 824 & 15.1 & 1,652 \\
\hline Some higher education & 730 & 13.5 & 428 & 7.6 & 1,158 \\
\hline Associate degree and above & 1,105 & 25.6 & 330 & 6.5 & 1,435 \\
\hline Marital status (all) & 3,302 & 64.1 & 2,095 & 35.9 & 5,397 \\
\hline Single/separated/widowed/divorced & 1,538 & 41.2 & 1,408 & 19.5 & 2,946 \\
\hline Married & 1,764 & 22.9 & 687 & 16.3 & 2,451 \\
\hline Parity (all) & 3,302 & 64.1 & 2,095 & 35.9 & 5,397 \\
\hline First infant & 521 & 9.1 & 344 & 5.2 & 865 \\
\hline 2 or more & 2,781 & 55.0 & 1,751 & 30.7 & 4,532 \\
\hline Preterm birth (all) & 3,254 & 64.1 & 2,068 & 35.9 & 5,322 \\
\hline Yes (less than 37 weeks) & 390 & 6.8 & 315 & 5.5 & 705 \\
\hline No (37 weeks and above) & 2,864 & 57.2 & 1,753 & 30.4 & 4,617 \\
\hline Low birth weight (all) & 3,302 & 64.1 & 2,095 & 35.9 & 5,397 \\
\hline Yes (less than 2500 grams) & 259 & 4.1 & 197 & 3.5 & 456 \\
\hline No (2500 grams and above) & 3,043 & 60.1 & 1,898 & 32.3 & 4,941 \\
\hline Insurance (all) & 3,302 & 64.1 & 2,095 & 35.9 & 5,397 \\
\hline Private or MediGap & 1,486 & 35.8 & 626 & 14.7 & 2,112 \\
\hline Medicaid, CHIP, or a state-sponsored plan & 779 & 9.8 & 821 & 11.0 & 1,600 \\
\hline Medicare, military, or other gv’t plans & 219 & 3.4 & 109 & 1.8 & 328 \\
\hline Single service plan, IHS, or no coverage & 818 & 15.2 & 539 & 8.4 & 1,357 \\
\hline Dwelling (all) & 3,302 & 64.1 & 2,095 & 35.9 & 5,397 \\
\hline Urban, principal city & 1,343 & 19.7 & 932 & 12.4 & 2,275 \\
\hline Urban, other than principal city & 1,585 & 36.1 & 780 & 17.0 & 2,365 \\
\hline Rural & 374 & 8.3 & 383 & 6.5 & 757 \\
\hline
\end{tabular}

Note: based on 5,397 participants with infants from the National Survey of Family Growth 2011-2013. Preterm data were missing for 75 participants and the number and percentage of breastfeeding by these participants are not presented in the table.

N: number; CHIP: Children's Health Insurance Program; gv't: government; IHS: Indian Health Service; Wt Col\%: weighted column percentage.

\section{Discussion}

The odds ratio for the association between FPL and breastfeeding failed to reach significance in an adjusted logistic regression with the covariates of maternal age, maternal race/ethnicity, maternal education, marital status, parity, preterm birth, low birth weight, insurance, and dwelling. Although studies exist which address only maternal low 
TABLE 2: Unadjusted odds ratios (OR) and 95\% confidence intervals (CI) of Federal Poverty Level (FPL) categories (reference group = 0-99\% FPL) from separate logistic regressions on breastfeeding National Survey of Family Growth (NSFG) 2011-2013.

\begin{tabular}{|c|c|c|c|c|c|c|}
\hline & Number & 100-199\% FPL & 200-299\% FPL & 300-399\% FPL & $400 \%+F P L$ & $\begin{array}{c}\text { Wald } \\
p \text { value }\end{array}$ \\
\hline All & 5,397 & $1.47(1.09,1.99)$ & $1.89(1.33,2.70)$ & $2.16(1.37,3.39)$ & $3.51(2.21,5.59)$ & $<0.001$ \\
\hline \multicolumn{7}{|l|}{ Maternal age } \\
\hline 14 to less than 20 years & 940 & $1.25(0.72,2.17)$ & $1.64(0.78,3.44)$ & $0.35(0.11,1.07)$ & $0.82(0.21,3.21)$ & 0.134 \\
\hline 20 to less than 25 years & 1,892 & $1.42(0.95,2.12)$ & $1.58(1.04,2.40)$ & $1.68(1.02,2.79)$ & $1.67(0.77,3.60)$ & 0.115 \\
\hline 25 to less than 30 years & 1,499 & $1.63(0.99,2.70)$ & $2.29(1.34,3.94)$ & $2.22(1.20,4.09)$ & $2.91(1.72,4.92)$ & $<0.001$ \\
\hline 30 to 44 years & 1,066 & $1.32(0.70,2.48)$ & $1.28(0.63,2.61)$ & $2.48(1.18,5.22)$ & $3.90(1.96,7.76)$ & 0.001 \\
\hline \multicolumn{7}{|l|}{ Maternal race/ethnicity } \\
\hline Non-Hispanic white & 2,337 & $1.89(1.18,3.03)$ & $2.57(1.60,4.12)$ & $2.78(1.62,4.78)$ & $4.18(2.36,7.40)$ & $<0.001$ \\
\hline Non-Hispanic black & 1,313 & $1.63(0.95,2.81)$ & $1.97(0.93,4.16)$ & $4.83(1.44,16.21)$ & $3.52(1.25,9.94)$ & 0.005 \\
\hline Hispanic & 1,747 & $1.16(0.62,2.01)$ & $1.05(0.40,2.76)$ & $0.78(0.28,2.17)$ & $2.95(1.27,6.86)$ & 0.023 \\
\hline \multicolumn{7}{|l|}{ Maternal education } \\
\hline Less than high school & 1,152 & $1.49(0.90,2.49)$ & $1.36(0.51,3.68)$ & $1.65(0.23,11.77)$ & $0.23(0.03,1.83)$ & 0.226 \\
\hline High school graduate & 1,652 & $1.47(0.86,2.50)$ & $1.73(0.83,3.62)$ & $1.26(0.46,3.43)$ & $1.59(0.73,3.46)$ & 0.448 \\
\hline Some higher education & 1,158 & $1.49(0.86,2.57)$ & $1.28(0.67,2.45)$ & $1.07(0.37,3.15)$ & $2.07(0.78,5.51)$ & 0.442 \\
\hline Associate degree and above & 1,143 & $0.91(0.40,2.07)$ & $1.71(0.87,3.36)$ & $2.03(0.94,4.36)$ & $2.51(1.24,5.07)$ & 0.018 \\
\hline \multicolumn{7}{|l|}{ Marital status } \\
\hline Single/separated/widowed/divorced & 2,946 & $1.28(0.84,1.97)$ & $1.50(0.87,2.60)$ & $1.62(0.81,3.22)$ & $2.16(1.06,4.38)$ & 0.153 \\
\hline Married & 2,451 & $1.39(0.77,2.51)$ & $1.55(0.95,2.53)$ & $1.68(0.85,3.33)$ & $2.99(1.49,6.00)$ & 0.044 \\
\hline \multicolumn{7}{|l|}{ Parity } \\
\hline First infant & 865 & $1.08(0.63,1.84)$ & $1.45(0.86,2.44)$ & $1.23(0.62,2.44)$ & $1.46(0.71,3.01)$ & 0.657 \\
\hline 2 or more & 4,532 & $1.54(1.11,2.14)$ & $1.96(1.32,2.92)$ & $2.43(1.41,4.21)$ & $4.29(2.59,7.09)$ & $<0.001$ \\
\hline \multicolumn{7}{|l|}{ Preterm birth } \\
\hline Yes (less than 37 weeks) & 705 & $1.36(0.97,1.90)$ & $1.77(1.13,2.79)$ & $1.98(1.09,3.60)$ & $3.34(1.96,5.69)$ & $<0.001$ \\
\hline No (37 weeks and above) & 4,617 & $1.65(1.06,2.59)$ & $2.15(1.32,3.48)$ & $2.61(1.37,4.96)$ & $3.75(2.06,6.83)$ & $<0.001$ \\
\hline \multicolumn{7}{|l|}{ Low birth weight } \\
\hline Yes (less than 2500 grams) & 456 & $1.32(0.58,2.98)$ & $1.50(0.44,5.15)$ & $2.25(0.68,7.44)$ & $4.62(1.70,12.54)$ & 0.036 \\
\hline No (2500 grams and above) & 4,941 & $1.46(1.07,2.00)$ & $1.94(1.39,2.72)$ & $2.13(1.34,3.38)$ & $3.40(2.11,5.49)$ & $<0.001$ \\
\hline \multicolumn{7}{|l|}{ Insurance } \\
\hline Private or MediGap & 2,112 & $1.62(0.94,2.81)$ & $1.57(0.89,2.77)$ & $1.80(1.03,3.16)$ & $3.62(1.94,6.76)$ & 0.001 \\
\hline Medicaid, CHIP, or a state-sponsored plan & 1,600 & $1.16(0.71,1.90)$ & $2.43(1.09,5.45)$ & $1.21(0.44,3.36)$ & $1.70(0.40,7.25)$ & 0.278 \\
\hline Medicare, military, or other gv't plans & 328 & $1.95(0.77,4.95)$ & $1.90(0.82,4.37)$ & $2.87(1.09,7.54)$ & $0.56(0.93,3.41)$ & 0.004 \\
\hline Single service plan, IHS, or no coverage & 1,357 & $1.08(0.62,1.88)$ & $2.19(0.99,4.85)$ & $3.77(1.55,9.16)$ & $1.64(0.72,3.71)$ & 0.004 \\
\hline \multicolumn{7}{|l|}{ Dwelling } \\
\hline Urban, principal city & 2,275 & $1.22(0.72,2.07)$ & $3.21(1.98,5.22)$ & $2.80(1.34,5.87)$ & $5.68(2.48,12.97)$ & $<0.001$ \\
\hline Urban, other than principal city & 2,365 & $1.95(1.31,2.90)$ & $1.54(0.94,2.52)$ & $1.87(1.01,3.48)$ & $3.11(1.70,5.70)$ & $<0.001$ \\
\hline Rural & 757 & $1.04(0.49,2.20)$ & $1.51(0.66,3.41)$ & $2.63(1.06,6.50)$ & $2.61(0.94,7.27)$ & 0.255 \\
\hline
\end{tabular}

Note: based on 5,397 mothers with infants from the National Survey of Family Growth 2011-2013. Preterm data were missing for 75 participants and the number and percentage of breastfeeding by these participants are not presented in the table.

The separate logistic regressions tested the relationship between breastfeeding and Federal Poverty Level categories of household income for each characteristic. CHIP: Children's Health Insurance Program; gv't: government; IHS: Indian Health Service; Wt Col\%: weighted column percentage.

FPL $=$ Federal Poverty Level.

0-99\% Federal Poverty Level is the reference group.

income and breastfeeding, there is a paucity of information concerning income disparity (concerning a more comprehensive and inclusive approach to income) and breastfeeding in the US. Nevertheless, similar results to support this study were found in a study of 10,519 women in California who gave birth between 1999 and 2001 in which Heck and colleagues [11] found that family income was not associated with breastfeeding. Lutter and Morrow [12] reported a trend in Africa, Asia, Latin America, and the Middle East over the previous two decades in which annual increases in breastfeeding were not associated with the gross national income of the participants' respective countries.

Researchers conducting a study of three hospitals in Canada found conflicting results regarding breastfeeding 
TABLE 3: Adjusted odds ratios (AOR) and 95\% confidence intervals (CI) from logistic regression on breastfeeding National Survey of Family Growth (NSFG) 2011-2013 ( $n=5397)$.

\begin{tabular}{|c|c|c|c|c|}
\hline Adjusted logistic regression & Model 1 & $p$ value & Model 2 & $p$ value \\
\hline \multicolumn{5}{|l|}{ Federal Poverty Level } \\
\hline 0-99\% of Federal Poverty Level & Reference & & Reference & \\
\hline $100-199 \%$ of Federal Poverty Level & $1.23(0.99,1.70)$ & 0.2091 & $1.19(0.86,1.65)$ & 0.305 \\
\hline $200-299 \%$ of Federal Poverty Level & $1.24(0.86,1.79)$ & 0.2392 & $1.25(0.88,1.76)$ & 0.212 \\
\hline $300-399 \%$ of Federal Poverty Level & $1.09(0.68,1.75)$ & 0.7081 & $1.05(0.65,1.70)$ & 0.831 \\
\hline $400 \%$ and above & $1.50(0.89,2.51)$ & 0.1281 & $1.45(0.83,2.52)$ & 0.193 \\
\hline \multicolumn{5}{|l|}{ Maternal age } \\
\hline 14 to less than 20 years & $0.76(0.57,1.00)$ & 0.0467 & $0.75(0.56,1.00)$ & 0.050 \\
\hline 20 to less than 25 years & Reference & & Reference & \\
\hline 25 to less than 30 years & $1.18(0.96,1.44)$ & 0.1180 & $1.17(0.95,1.43)$ & 0.137 \\
\hline 30 to 44 years & $1.56(1.15,2.13)$ & 0.0008 & $1.61(1.18,2.18)$ & 0.002 \\
\hline \multicolumn{5}{|l|}{ Maternal race/ethnicity } \\
\hline Non-Hispanic white & & & Reference & \\
\hline Non-Hispanic black & & & $0.52(0.38,0.71)$ & 0.004 \\
\hline Hispanic & & & $1.47(1.03,2.08)$ & 0.516 \\
\hline \multicolumn{5}{|l|}{ Maternal education } \\
\hline Less than high school & Reference & & Reference & \\
\hline High school graduate & $0.53(0.35,0.79)$ & 0.0019 & $0.61(0.39,0.98)$ & 0.039 \\
\hline Some higher education & $0.88(0.59,1.33)$ & 0.5527 & $1.10(0.69,1.76$ & 0.693 \\
\hline Associate degree and above & $1.50(0.99,2.27)$ & 0.0569 & $1.87(1.16,3.00)$ & 0.010 \\
\hline \multicolumn{5}{|l|}{ Marital status } \\
\hline Single/separated/widowed/divorced & $0.65(0.50,0.84)$ & & $0.89(0.73,1.083)$ & 0.221 \\
\hline Married & Reference & & Reference & \\
\hline \multicolumn{5}{|l|}{ Parity } \\
\hline First infant & & & Reference & \\
\hline 2 or more & & & $0.91(0.71,1.16)$ & 0.432 \\
\hline \multicolumn{5}{|l|}{ Low birth weight } \\
\hline Yes (less than 2500 grams) & & & $0.86(0.55,1.33)$ & 0.486 \\
\hline No (2500 grams and above) & & & Reference & \\
\hline \multicolumn{5}{|l|}{ Preterm birth } \\
\hline Yes(less than 37 weeks) & & & Reference & \\
\hline No (37 weeks and above) & & & $1.21(0.93,1.57)$ & 0.164 \\
\hline \multicolumn{5}{|l|}{ Insurance } \\
\hline Private or MediGap & & & $0.85(0.60,1.22)$ & 0.383 \\
\hline Medicaid, CHIP, or a state-sponsored plan & & & $0.61(0.44,0.85)$ & 0.003 \\
\hline Medicare, military, or other government plans & & & $1.02(0.68,1.55)$ & 0.909 \\
\hline Single service plan, Indian Health Service, or no coverage & & & Reference & \\
\hline \multicolumn{5}{|l|}{ Dwelling } \\
\hline Urban, principal city & $1.56(1.06,2.29)$ & 0.0243 & $1.62(1.07,2.46)$ & 0.023 \\
\hline Urban, other than principal city & $1.55(1.11,2.17)$ & 0.0103 & $1.48(1.04,2.09)$ & 0.028 \\
\hline Rural & Reference & & Reference & \\
\hline
\end{tabular}

Note: based on 5,397 participants with infants from the National Survey of Family Growth 2011-2013.

initiation and maternal income. Overall breastfeeding initiation increased over time, although for one hospital the rate difference between maternal high income and low income decreased, for another hospital the rate difference remained the same, and for the third hospital the rate difference increased [15].

Supporting the association between breastfeeding rates and income, researchers using the 1999-2006 National 
TABLE 4: Adjusted stratified logistic regression results with race/ethnicity, gestation, and marital status on breastfeeding, National Survey of Family Growth (NSFG) 2011-2013.

\begin{tabular}{|c|c|c|c|c|c|c|}
\hline & \multicolumn{6}{|c|}{ Adjusted odds ratios ( $95 \%$ confidence interval) } \\
\hline & Number & $100-199 \%$ FPL & $200-299 \%$ FPL & $300-399 \%$ FPL & $400 \%+F P L$ & $p$ value \\
\hline \multicolumn{7}{|l|}{ Maternal race/ethnicity } \\
\hline Non-Hispanic white $^{1}$ & 2,337 & $1.01(0.61,1.70)$ & $0.93(0.53,1.61)$ & $0.83(0.44,1.59)$ & $1.00(0.52,1.92)$ & 0.974 \\
\hline Non-Hispanic black ${ }^{2}$ & 1,313 & $1.23(0.80,1.90)$ & $1.67(0.90,3.11)$ & $1.96(0.79,4.88)$ & $1.81(0.84,3.91)$ & 0.253 \\
\hline Hispanic $^{3}$ & 1,747 & $1.17(0.62,2.19)$ & $1.21(0.47,3.09)$ & $0.83(0.39,1.76)$ & $2.49(0.92,6.76)$ & 0.219 \\
\hline \multicolumn{7}{|l|}{ Marital status } \\
\hline Single/separated/widowed/divorced ${ }^{4}$ & 2,946 & $1.15(0.79,1.68)$ & $1.28(0.71,2.32)$ & $1.18(0.54,2.56)$ & $1.28(0.58,2.84)$ & 0.892 \\
\hline Married $^{5}$ & 2,451 & $1.16(0.64,2.09)$ & $0.94(0.53,1.66)$ & $0.90(0.43,1.87)$ & $1.18(0.58,2.37)$ & 0.869 \\
\hline \multicolumn{7}{|l|}{ Preterm birth } \\
\hline Yes (less than 37 weeks) ${ }^{6}$ & 705 & $1.14(0.64,2.04)$ & $1.14(0.66,1.98)$ & $1.13(0.51,2.53)$ & $1.44(0.66,3.13)$ & 0.918 \\
\hline No (37 weeks and above) $)^{7}$ & 4,617 & $1.15(0.85,1.58)$ & $1.26(0.82,1.93)$ & $0.98(0.56,1.73)$ & $1.40(0.72,2.75)$ & 0.759 \\
\hline \multicolumn{7}{|l|}{ Interactions } \\
\hline Non-Hispanic white, married, not preterm & 544 & $0.60(0.20,1.75)$ & $0.64(0.17,2.34)$ & $0.64(0.16,2.61)$ & $0.81(0.19,3.46)$ & 0.864 \\
\hline Non-Hispanic white, single, not preterm & 311 & $0.86(0.39,1.90)$ & $0.84(0.31,2.32)$ & $0.32(0.08,1.27)$ & $0.43(0.09,2.08)$ & 0.441 \\
\hline Non-Hispanic white, married, preterm & 857 & $1.12(0.39,3.22)$ & $0.56(0.20,1.59)$ & $0.71(0.22,2.32)$ & $0.86(0.25,2.95)$ & 0.866 \\
\hline Non-Hispanic white, single, preterm & 625 & $1.08(0.57,2.04)$ & $3.00(1.39,6.48)$ & $1.61(0.54,4.83)$ & $1.08(0.37,3.12)$ & 0.086 \\
\hline Non-Hispanic black, married, not preterm & 89 & $0.76(0.02,27.42)$ & $1.51(0.01,141.84)$ & $1.89(0.04,91.30)$ & $1.03(0.01,113.05)$ & 0.987 \\
\hline Non-Hispanic black, single, not preterm & 334 & $1.37(0.45,4.12)$ & $0.76(0.20,2.86)$ & $7.02(0.79,62.55)$ & $0.72(0.13,4.02)$ & 0.232 \\
\hline Non-Hispanic black, married, preterm & 208 & $2.09(0.55,7.93)$ & $2.43(0.49,12.01)$ & $14.20(1.61,125.07)$ & $6.08(0.66,55.59)$ & 0.083 \\
\hline Non-Hispanic black, single, preterm & 682 & $1.42(0.76,2.66)$ & $2.28(0.79,6.59)$ & $1.38(0.38,4.99)$ & $2.04(0.50,8.36)$ & 0.547 \\
\hline Hispanic, married, not preterm & 204 & Limited cell sizes & Limited cell sizes & Limited cell sizes & Limited cell sizes & \\
\hline Hispanic, single, not preterm & 250 & $0.83(0.33,2.08)$ & $0.07(0.01,0.36)$ & Limited cell sizes & Limited cell sizes & \\
\hline Hispanic, married, preterm & 549 & $0.82(0.50,1.32)$ & $1.60(0.48,5.35)$ & $0.29(0.07,1.29)$ & $1.11(0.27,4.58)$ & 0.327 \\
\hline Hispanic, single, preterm & 744 & $1.04(0.43,2.50)$ & $2.02(0.59,6.92)$ & $1.15(0.28,4.74)$ & $1.42(0.27,7.42)$ & 0.834 \\
\hline
\end{tabular}

FPL $=$ Federal Poverty Level.

0-99\% Federal Poverty Level is the reference group.

Subgroups are adjusted for maternal age (14 to less than 20 years; 20 to less than 25 years; 25 to less than 30 years; or 30 to 44 years), maternal race/ethnicity (non-Hispanic white; non-Hispanic black; or Hispanic), maternal education (less than high school; high school graduate; some higher education; or Associate degree and above), marital status (married or single/separated/divorced), parity (first infant or 2 or more), preterm birth (yes, less than 37 weeks, or no, 37 weeks and above), low birth weight (yes, less than 2500 grams, or no, 2500 grams and above), insurance (private or MediGap; Medicaid, CHIP, or a statesponsored plan; Medicare, military, or other government plans; or single service plan, IHS, or no coverage), and dwelling (urban, principal city; urban, other than principal city; or rural) except as noted.

${ }^{1}$ Stratified to non-Hispanic white participants. ${ }^{2}$ Stratified to non-Hispanic black participants. ${ }^{3}$ Stratified to Hispanic participants. ${ }^{4}$ Stratified to single/separated/widowed/divorced participants. ${ }^{5}$ Stratified to married participants. ${ }^{6}$ Stratified to participants with preterm infants. ${ }^{7}$ Stratified to participants with infants who were not preterm.

Health and Nutrition Examination Surveys suggested that women with higher incomes were significantly more likely to breastfeed than women with low incomes [13]. In a large population-based ecological study in Ontario, Canada, women with higher incomes were more likely to breastfeed their infants than women with lower incomes [14].

Future studies are needed to further clarify the role of FPL on breastfeeding and to examine the role of supportive services, such as WIC, in encouraging breastfeeding. This study's main limitation is that the data collected were selfreported. Self-reported measures are subject to social desirability bias, which occur when a participant responds in a way to appear more positive. Second, a causal interpretation cannot be applied as the cross-sectional design of the study does not indicate temporality. However, the study has several strengths. It is a large study using national data and the sampling used a multistage probability-based and representative design.

\section{Conclusion}

While FPL was significantly associated with lack of breastfeeding in unadjusted analyses, the role of FPL failed to reach significance in the adjusted regression analyses of the study, suggesting a need for all women to have breastfeeding initiation support. Women of childbearing age should be educated about the benefits of breastfeeding for themselves and their infants. The workers in supportive programs, such as WIC, are important in encouraging breastfeeding in their clients. Healthcare providers should continue to promote breastfeeding and to help meet Healthy People 2020 goals.

\section{Disclaimer}

The content is solely the responsibility of the authors and does not necessarily represent the official views of the National Institutes of Health. 


\section{Conflict of Interests}

The authors declare that there is no conflict of interests regarding the publication of this paper.

\section{Acknowledgment}

Research in this publication was supported by the National Institute of General Medical Sciences of the National Institutes of Health under Award nos. U54GM104942; WVCTSI; R01 DE014899.

\section{References}

[1] A. I. Eidelman and R. J. Schanler, "Policy statement. Breastfeeding and the use of human milk," Pediatrics, vol. 129, no. 3, pp. e827-e841, 2012.

[2] Healthy People 2020, "Maternal, Infant, and Child Health 2020 Topics \& Objectives," 2015, http://www.healthypeople.gov/2020/ topics-objectives/topic/maternal-infant-and-child-health/objectives.

[3] L. M. Dinour, G. A. Pope, and Y. K. Bai, "Breast milk pumping beliefs, supports, and barriers on a university campus," Journal of Human Lactation, vol. 31, no. 1, pp. 156-165, 2015.

[4] A. Dixit, L. Feldman-Winter, and K. A. Szucs, "Frustrated,' "depressed," and "devastated" pediatric trainees: US academic medical centers fail to provide adequate workplace breastfeeding support," Journal of Human Lactation, vol. 31, no. 2, pp. 240248, 2015.

[5] V. J. Flaherman, K. G. Hicks, J. Huynh, M. D. Cabana, and K. A. Lee, "Positive and negative experiences of breast pumping during the first 6 months," Maternal and Child Nutrition, 2014.

[6] C. T. Besore, "Barriers to breastfeeding for hispanic mothers," Breastfeeding Medicine, vol. 9, no. 7, pp. 352-354, 2014.

[7] X. Ma, J. Liu, and M. Smith, "WIC participation and breastfeeding in south carolina: updates from PRAMS 2009-2010," Maternal and Child Health Journal, vol. 18, no. 5, pp. 1271-1279, 2014.

[8] I. C. Hedberg, "Barriers to breastfeeding in the WIC population," The American Journal of Maternal Child Nursing, vol. 38, no. 4, pp. 244-249, 2013.

[9] T. T. Gross, R. Powell, A. K. Anderson, J. Hall, M. Davis, and K. Hilyard, "WIC peer counselors' perceptions of breastfeeding in African American women with lower incomes," Journal of Human Lactation, vol. 31, no. 1, pp. 99-110, 2015.

[10] E. Jensen, "Participation in the supplemental nutrition program for women, infants and children (WIC) and breastfeeding: national, regional, and state level analyses," Maternal and Child Health Journal, vol. 16, no. 3, pp. 624-631, 2012.

[11] K. E. Heck, P. Braveman, C. Cubbin, G. F. Chávez, and J. L. Kiely, "Socioeconomic status and breastfeeding initiation among california mothers," Public Health Reports, vol. 121, no. 1, pp. 51-59, 2006.

[12] C. K. Lutter and A. L. Morrow, "Protection, promotion, and support and global trends in breastfeeding," Advances in Nutrition, vol. 4, pp. 213-219, 2013.

[13] M. M. McDowell, C. Y. Wang, and J. Kennedy-Stephenson, Breastfeeding in the United States: Findings from the National Health and Nutrition Examination Surveys, 1999-2006, U.S. Department of Health and Human Services, Centers for Disease
Control and Prevention, National Center for Health Statistics, 2008.

[14] S. D. McDonald, E. Pullenayegum, B. Chapman et al., "Prevalence and predictors of exclusive breastfeeding at hospital discharge," Obstetrics \& Gynecology, vol. 119, no. 6, pp. 1171-1179, 2012.

[15] N. C. Nickel, P. J. Martens, D. Chateau et al., "Have we left some behind? Trends in socio-economic inequalities in breastfeeding initiation: a population-based epidemiological surveillance study," Canadian Journal of Public Health, vol. 105, no. 5, pp. e362-e368, 2014.

[16] CDC, National Survey of Family Growth. 2011-2013 NSFG: Public Use Data Files, Codebooks, and Documentation, 2015, http://www.cdc.gov/nchs/nsfg/nsfg_2011_2013_puf.htm. 


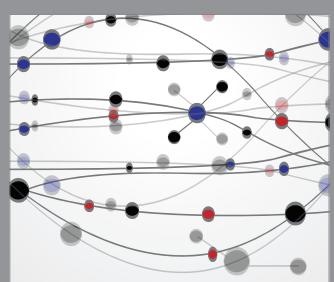

The Scientific World Journal
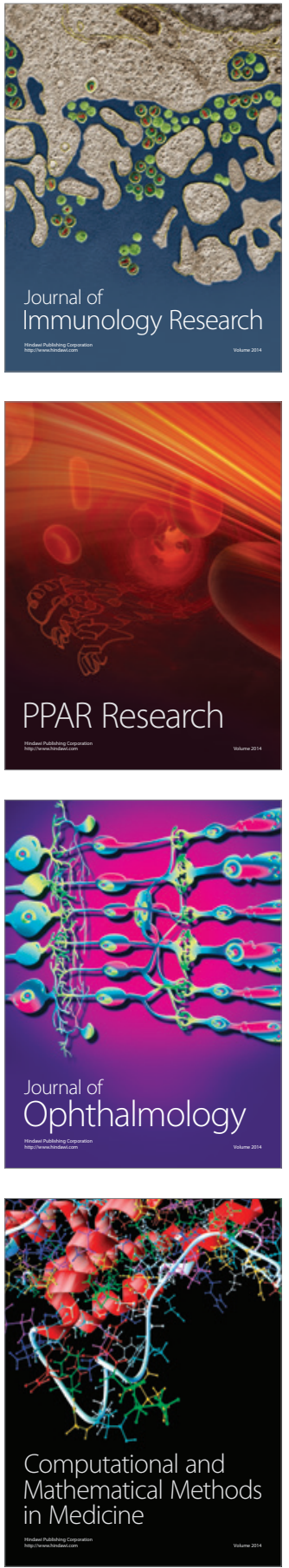

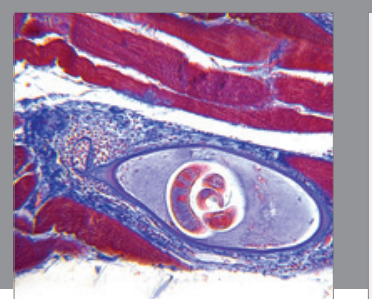

Gastroenterology Research and Practice

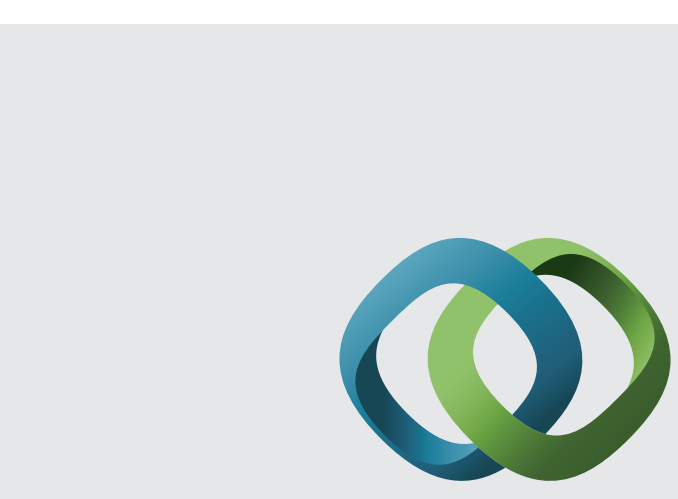

\section{Hindawi}

Submit your manuscripts at

http://www.hindawi.com
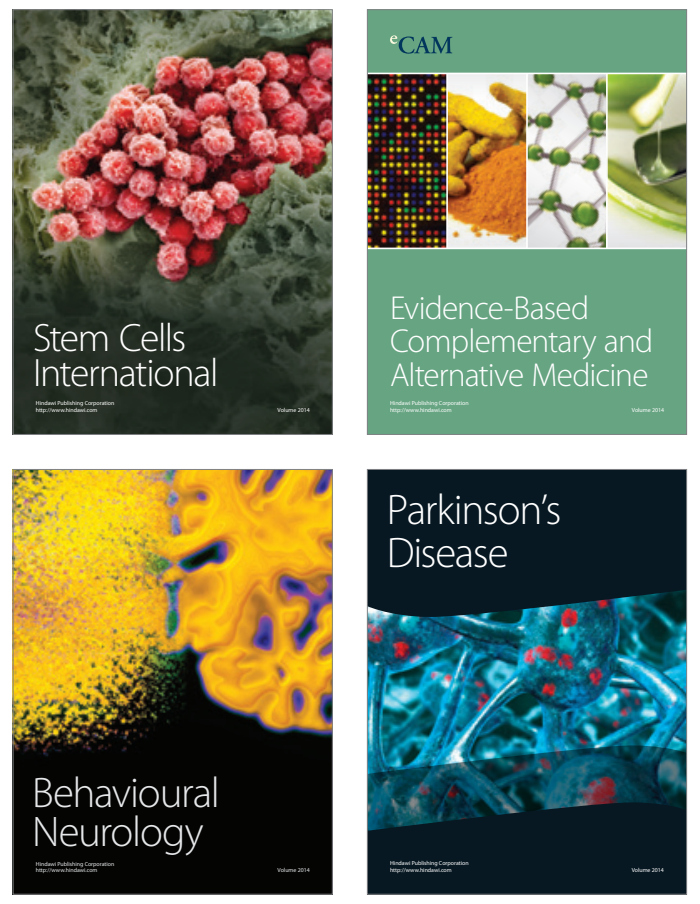
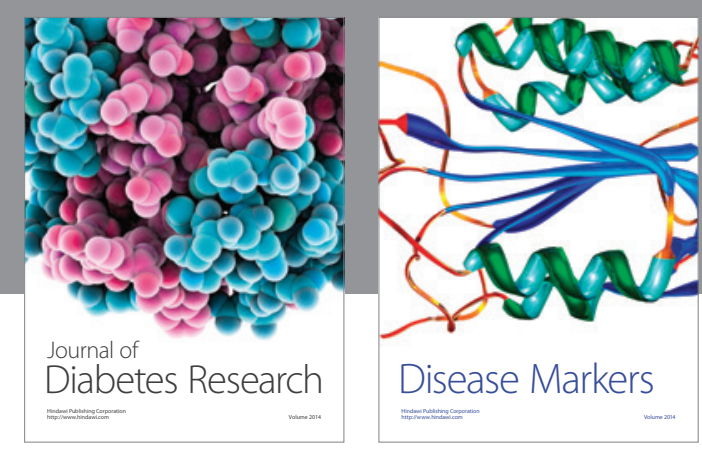

Disease Markers
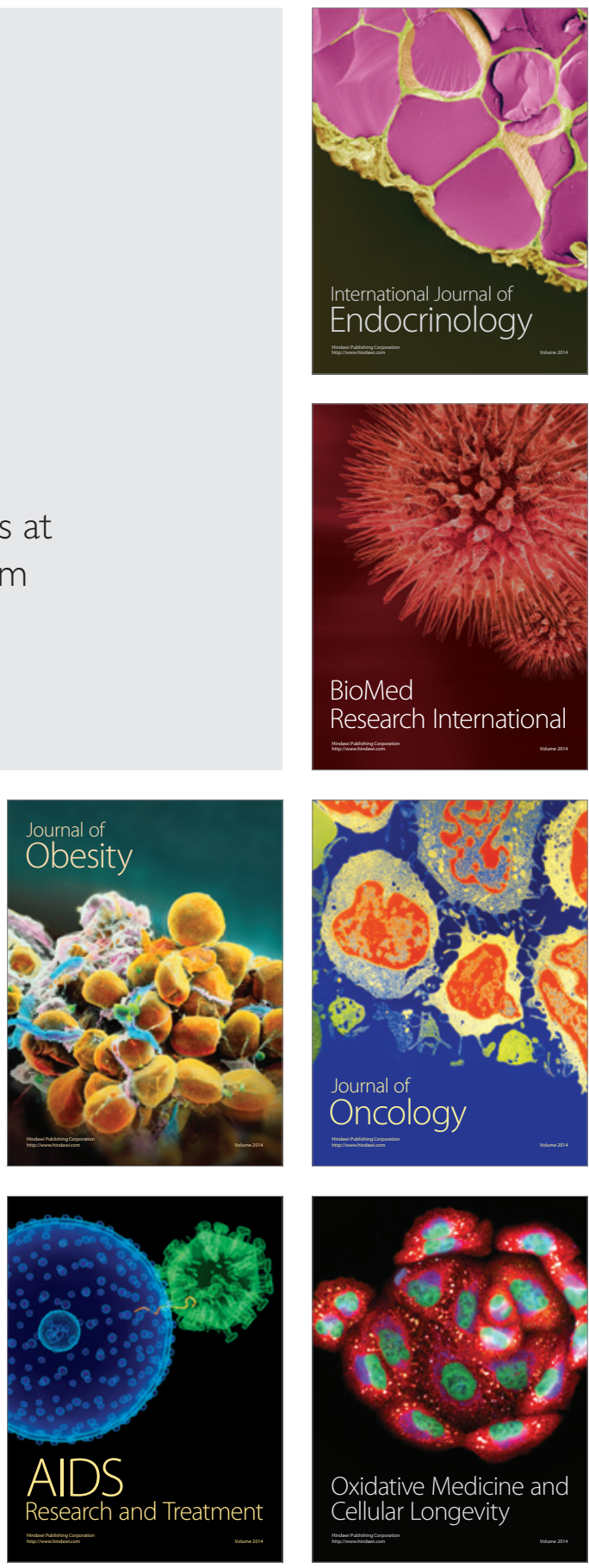